OPEN ACCESS

Edited by:

Vivian Afi Abui Dzokoto,

Virginia Commonwealth University,

United States

Reviewed by:

Mary Alice Varga,

University of West Georgia,

United States

Amit Shrira,

Bar-Ilan University, Israel

${ }^{*}$ Correspondence:

Ningning Zhou

zhouningning1990@mail.bnu.edu.cn

Specialty section:

This article was submitted to

Cultural Psychology,

a section of the journal

Frontiers in Psychology

Received: 03 September 2019 Accepted: 16 December 2019

Published: 10 January 2020

Citation:

Stelzer E-M, Zhou N, Maercker A, O'Connor M-F and Killikelly C (2020)

Prolonged Grief Disorder and the Cultural Crisis.

Front. Psychol. 10:2982.

doi: 10.3389/fpsyg.2019.02982

\section{Prolonged Grief Disorder and the Cultural Crisis}

\author{
Eva-Maria Stelzer ${ }^{1,2}$, Ningning Zhou ${ }^{1,3 *}$, Andreas Maercker ${ }^{1}$, Mary-Frances O'Connor ${ }^{2}$ \\ and Clare Killikelly ${ }^{1}$
}

' Division of Psychopathology and Clinical Intervention, University of Zurich, Zurich, Switzerland, ${ }^{2}$ Department of Psychology, University of Arizona, Tucson, AZ, United States, ${ }^{3}$ Faculty of Psychology, Beijing Normal University, Beijing, China

Prolonged grief disorder (PGD) is included as a new mental health disorder in the 11th edition of the International Classification of Diseases (ICD-11). Understandably, this has boosted research efforts to investigate this newcomer to psychopathology. However, the use of different diagnostic algorithms has resulted in substantially different prevalence rates both within and across cultural groups. Furthermore, global applicability of the new criteria outside of the Global North has not been yet been established. This perspective presents key findings from Asian research groups and discusses the roadblocks to unified PGD research, including the heterogeneric use of diagnostic algorithms and the lack of cultural compatibility of ICD-11 items. The authors discuss the key issues and address implications for practice.

\section{Keywords: prolonged grief disorder, Asian, cross-culture, prevalence, bereavement}

\section{HIGHLIGHTS}

- The new ICD-11 Prolonged Grief Disorder (PGD) criteria are conceptualized in terms of the World Health Organization's prioritization of international applicability.

- However, significant barriers exist to extrapolate prevalence rates within and across culture, and to establish global applicability.

- Barriers to unified PGD research include, among others, the use of different diagnostic algorithms and a lack of research regarding the cultural specificity of current ICD-11 items leading to an evolving cultural crisis of potential misestimation of prevalence rates.

- Emerging research from Asia confirms the worldwide heterogeneity in the use of PGD algorithms and the extent of the crisis.

\section{INTRODUCTION}

The field of grief and bereavement is at a turning point. For the first time, prolonged grief disorder (PGD) is included as a new mental health disorder in the World Health Organization's (WHO) 11th edition of the International Classification of Diseases (ICD-11) (World Health Organization [WHO], 2018). This presents the opportunity for igniting research into assessment 
and treatment and providing clinicians with strong and unified groundwork for the validity of this disorder. However, currently there are some significant roadblocks to concerted PGD research. Firstly, the new formulation of PGD is preceded by several different disorder definitions that are currently in use. This has led to a wide variation in the use of diagnostic algorithms yielding substantially different prevalence rates, also across different cultural groups. This is unaided by the fact that the ICD-11 has not formally included a recommended diagnostic algorithm but rather applies a typological approach for diagnoses. Secondly, the clinical description of PGD for the ICD-11 is newly conceptualized following the WHO's prioritization of global applicability. PGD is characterized by core symptoms of longing for or preoccupation with deceased, significant symptoms of emotional distress, in addition to key cultural caveats, i.e., the duration of disorder must violate expected social and cultural norms (Killikelly and Maercker, 2017; World Health Organization [WHO], 2018). However, the global applicability of diagnostic criteria outside of the Global North has not yet been established. For example, the content of the specific items is largely derived from the Global North (Prigerson et al., 2009; Maciejewski et al., 2016). Several research groups across Asia are spearheading a new wave of research that is exploring and evaluating PGD. This perspective presents the latest research from Asia that uses the PGD criteria (as opposed to complicated grief or persistent complex bereavement disorder) and the new ICD-11 formulation of PGD. Here we present key findings from EastAsian research groups that expose the widespread difficulty with the heterogenic use of diagnostic algorithms and also challenge surface-level cultural compatibility of the specific ICD-11 items. We propose that the continued use of different diagnostic algorithms will undermine the field and lead to a crisis in terms of inaccurate prevalence rates of PGD, particularly in cross cultural research.

\section{PREVALENCE CRISIS}

Around the world, prevalence rates for PGD have been examined using several different sets of diagnostic algorithms. Most predominantly the PGDPlos criteria (Prigerson et al., 2009), ICD-11 PGD criteria (as closely as possible modeled after ICD-11; Maciejewski et al., 2016; Killikelly and Maercker, 2017), criteria from the Inventory of Complicated Grief (ICG) (Prigerson et al., 1995; Prigerson and Maciejewski, 2005; Shear et al., 2005), and variations of these criteria are used (Table 1). Not surprisingly, the use of different criteria sets has resulted in different prevalence rates of diagnosis. Using the PGDPlos criteria (Prigerson et al., 2009), prevalence rates for PGD in East Asian studies range from $1.8 \%$ for the general population ( $\mathrm{He}$ et al., 2014) to $21.73 \%$ for those who lost their only child (Shi et al., 2019). In contrast, a recent study that applied the new ICD-11 PGD criteria following Killikelly and Maercker (2017) and Maciejewski et al. (2016) documents prevalence rates of 38.7 and $31.5 \%$ for Chinese Shidu parents (Zhou et al., under review). Finally, a range of other variations on these criteria have revealed different prevalence rates. For instance, studies operationalizing PGD in terms of the CG formulation (Prigerson et al., 1995; Prigerson and Maciejewski, 2005; Shear et al., 2005) find prevalence rates ranging from 9.8 (Tsutsui et al., 2014) to 35\% (Yu et al., 2016). And according to Yu et al. (2017) almost half of their sample (47.2) met criteria for PGD applying yet another diagnostic algorithm to dichotomize individuals in PGD vs. non-PGD clusters.

A similar variation can be found for research conducted in the Global North (Bonanno and Malgaroli, 2019; O'Connor et al., 2019). For instance, in a sample of US widow(er)s, Bonanno and Malgaroli (2019) reported prevalence rates of 4.8-12.2\% when comparing different diagnostic algorithms for ICD-11 PGD criteria with varying numbers of accessory symptom items. The use of these different diagnostic algorithms makes it very difficult to extrapolate prevalence rates of PGD across Asian countries and to compare these rates with prevalence rates in the Global North, posing a significant risk of over- or underestimating the rate of disorder. For instance, a higher diagnostic threshold for assessing pathological grief has been suggested for Chinese samples (Li and Prigerson, 2016). However, this may only be the case when using the CG formulation applied by Li and Prigerson (2016). Furthermore, comparability and conclusions are limited as algorithms are derived from a number of scales, assessment procedures (self-report vs. clinical interviews), and item responses (score of at least three vs. four) to assess symptom endorsement.

\section{BEYOND PREVALENCE}

In addition, it is unclear if the prevalence rates indicate the most valid symptom expression. Although in both Chinese individuals and samples from the Global North, yearning or longing for the deceased, indicative of separation-related distress, constitute the hallmark symptom for disordered grief (Prigerson et al., 2009; He et al., 2014; Li and Prigerson, 2016; Xiu et al., 2016), there may be other culturally bound symptoms of grief that are currently not included in the ICD-11 PGD criteria (Killikelly et al., 2018). There are several examples of how mental health measurement derived from within the cultural group may inform the validity of the assessment. In a foundational series of studies, Hinton et al. (2013) explored symptoms of PGD in Cambodian refugees using a gold standard grief scale from the Global North [Prolonged Grief-13 (PG-13), Prigerson et al., 2009]. Interestingly they also developed a culturally sensitive measure of grief (CSM-G) and compared the rates of distress using these two scales. The CSM-G measure included reference to the somatic and bodily experiences of distress that are not included in the PG-13. According to the PG-13, 8\% of participants experienced significant grief distress, whereas the locally derived scale revealed that over $31 \%$ of participants met criteria for severe distress. These contrasting results between etic (measures developed outside of the culture) and emic (measures developed from within the culture) scales have been consistently found across different 
TABLE 1 | Common diagnostic algorithms for the assessment of PGD and their prevalence rates in East-Asian samples.

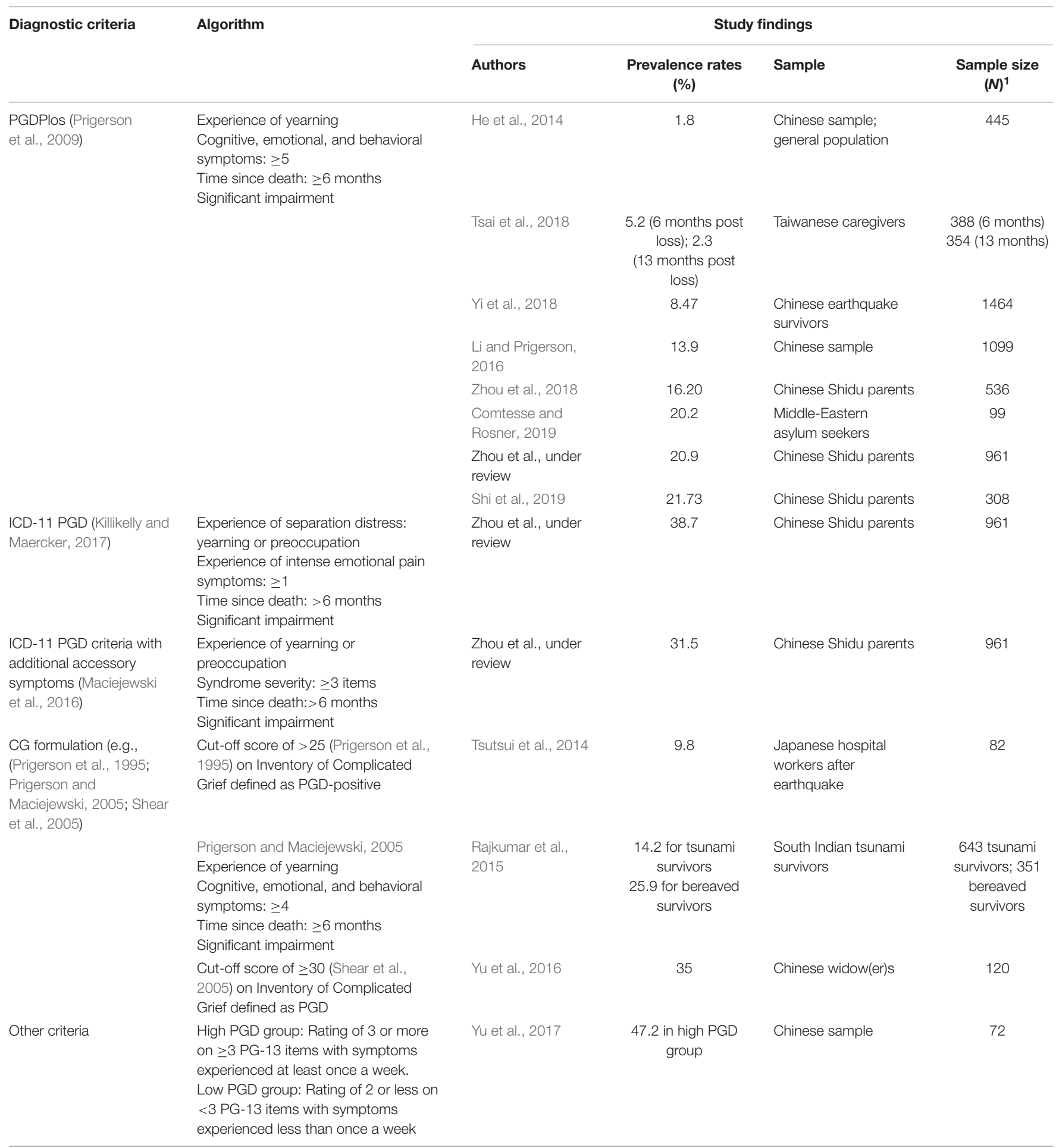

1 If information was available, the number of participants used for prevalence estimation is presented (rather than entire sample size of a particular study).

cultural groups and for different mental health disorders (Patel et al., 1997; Fernando, 2012; Rasmussen et al., 2014; Kim et al., 2017). If the ICD-11 guidelines for PGD seek global applicability, guidance on specific adaptations for different cultural groups should be provided to improve the cross-cultural validity (Bäärnhielm et al., 2015; Lewis-Fernández et al., 2017; Smid et al., 2018).

Additionally, features of the ICD-11 PGD criteria may be more or less common for certain cultural subgroups and thus should be weighted accordingly. We explored the 
cross-cultural utility and applicability of the ICD-11 PGD criteria among Chinese and German-speaking health-care providers (Stelzer et al., in press). While health-care providers were generally aligned with the diagnostic criteria, they also identified symptoms currently missing in the ICD-11 such as the experience of somatic/physical symptoms after loss, and symptoms that should be removed from the diagnostic criteria for certain cultural groups (e.g., anger, sadness, or loss of self in China). Cultural variations also became apparent for the time-criterion. Here, Chinese health-care providers suggested to extend the criterion to match the culturally prescribed mourning period. Such differences between the Global North and Asian samples with regard to endorsement of specific accessory symptoms have also been noted in quantitative studies ( $\mathrm{Li}$ and Prigerson, 2016; Xiu et al., 2016). In a cross-cultural comparison of bereaved parents, the Chinese sample reported a stronger sense that life is empty or meaningless whereas Swiss parents suffered from more severe grief-related preoccupation (Xiu et al., 2016). Similarly, trauma-related distress and grief hallucinations are particularly common in Chinese samples ( $\mathrm{Li}$ and Prigerson, 2016) but less strongly endorsed in the Global North. And of course, sociocultural and logistical barriers such as a lack of culturesensitive assessment tools and unfamiliarity with diagnostic systems further challenge the global applicability of ICD-11 PGD criteria (Stelzer et al., in press). Overall, these findings highlight the need to consider cultural factors and specific symptoms when assessing and generalizing PGD criteria across cultural groups.

\section{DISCUSSION}

Many cultural factors, working invisibly in the background, affect the way symptoms are expressed, perceived, assessed, interpreted, and documented. Religious beliefs and practices, for instance, are strongly related to culture and may account for some of the variations in grieving processes (Lobar et al., 2006; Beyers, 2017). Influenced by beliefs such as fatalism [e.g., "Life and death are decreed by fate" (Yang, 1980)], Chinese bereaved seldomly share their grief with others and when Chinese people comfort the bereaved they often do so by saying "Restrain your grief and accord with inevitable changes". The subtle influences of cultural context are observable when someone outside the culture disrupts the invisible veil that hides them. This brings to light important cultural differences that have not been discussed in the scientific literature. As empirical research forms the basis for clinical decision-making, validation of grief reactions across cultures is essential prior to generalizing symptom clusters - especially when trying to provide adequate support and comfort to the bereaved. The initial period when guidelines are first applied by clinicians is critical - before years of practice have solidified them, making them more resistant to change. Since the expression of grief varies by culture, despite the universal experience of grief, it is vitally important that we detect these variations early as we apply diagnostic criteria. In recent years, an increasing number of empirical studies (of Chinese origin in particular) have explored grief responses in samples beyond the Global North and added to a growing body of knowledge regarding prevalence rates of pathological grief. But despite these efforts from researchers and WHO stakeholders, we are far from establishing global applicability of PGD. The heterogeneity of diagnostic algorithms used by researchers and clinicians resulting in different prevalence rates within and across cultural groups constitutes one of the major challenges of cultural compatibility. If researchers keep using different diagnostic approaches to assess cases of PGD, conclusions and comparisons regarding prevalence rates and cross-cultural applicability are hampered.

We therefore recommend that expert researchers and clinicians in the field come together to decide on a consensus PGD algorithm that can be used across research groups and countries. The new wave of research emerging from Asian countries should stimulate collaboration to explore crosscultural similarities and differences in the presentation of grief. With the novum of having disordered grief included in classification systems for mental health disorders, it is critical to no longer presume a universality of grief responses but instead move toward a culturally integrated grief framework. Further research can examine this by collecting open-ended written responses to questions or interviews about grief reactions in a culture and generating culture-specific grief items (Shoeb et al., 2007; Yin et al., 2017). Additionally, cultural clinical interviews could be employed to grasp a full understanding about grief severity. For example, recently, researchers in the Netherlands have developed the Grief and Bereavement cultural interview that aims to provide an indepth assessment of the cultural context underlying prolonged grief distress (Smid et al., 2018). This 10-question interview could supplement a standard grief scale such as the PG13 or the new International PGD scale (Killikelly et al., in preparation) to better guide the severity of the diagnosis and treatment planning.

Although there is no gold standard procedure for crosscultural measurement of PGD and it is currently unknown how feasible such an assessment might be for grief, several researchers have confirmed that culturally informed mental health assessment is feasible, useful, and improves the validity of mental health diagnosis (Aggarwal et al., 2014; Kirmayer et al., 2014; Lewis-Fernández et al., 2017). Methodologically sound investigations and systematic cross-cultural comparisons can help identify in what ways grief phenomena are universal and culture specific. This knowledge, in turn, can influence existing bereavement models and clinical practice allowing researchers and clinicians to take individuals' cultural identities into account when providing assistance and comfort to culturally diverse populations. It is important for researchers and clinicians to share knowledge and develop a consensus on required cultural caveats and culturally specific symptoms to improve assessment and treatment of disordered grief. 


\section{AUTHOR CONTRIBUTIONS}

E-MS constructed the structure of the perspective and wrote most of it. NZ searched the articles conducted in

\section{REFERENCES}

Aggarwal, N. K., Glass, A., Tirado, A., Boiler, M., Nicasio, A., Alegría, M., et al. (2014). The development of the DSM-5 cultural formulation interview-fidelity instrument (CFI-FI): a pilot study. J. Health Care Poor Underserved 25:1397. doi: 10.1353/hpu.2014.0132

Bäärnhielm, S., Åberg Wistedt, A., and Rosso, M. S. (2015). Revising psychiatric diagnostic categorisation of immigrant patients after using the cultural formulation in DSM-IV. Transcult. Psychiatry 52, 287-310. doi: 10.1177/ 1363461514560657

Beyers, J. (2017). Religion and culture: revisiting a close relative. HTS Theo. Stud. 73, 1-9. doi: 10.4102/hts.v73i1.3864

Bonanno, G. A., and Malgaroli, M. (2019). Trajectories of grief: comparing symptoms from the DSM-5 and ICD-11 diagnoses. Depress. Anxiety. doi: 10. 1002/da.22902

Comtesse, H., and Rosner, R. (2019). Prolonged grief disorder among asylum seekers in Germany: the influence of losses and residence status. Eur. J. Psychotraumatol. 10:1591330. doi: 10.1080/20008198.2019.1591330

Fernando, G. A. (2012). The roads less traveled: mapping some pathways on the global mental health research roadmap. Transcult. Psychiatry 49, 396-417. doi: $10.1177 / 1363461512447137$

He, L., Tang, S., Yu, W., Xu, W., Xie, Q., and Wang, J. (2014). The prevalence, comorbidity and risks of prolonged grief disorder among bereaved Chinese adults. Psychiatry Res. 219, 347-352. doi: 10.1016/j.psychres.2014.05.022

Hinton, D. E., Nickerson, A., and Bryant, R. A. (2013). Prolonged grief in Cambodian refugees following genocide: rebirth concerns and avoidance of reminders. J. Loss Trauma 18, 444-460. doi: 10.1080/15325024.2012.714218

Killikelly, C., Bauer, S., and Maercker, A. (2018). The assessment of grief in refugees and post-conflict survivors: a narrative review of etic and emic research. Front. Psychol. 22:1957. doi: 10.3389/fpsyg.2018.01957

Killikelly, C., and Maercker, A. (2017). Prolonged grief disorder for ICD11: the primacy of clinical utility and international applicability. Eur. J. Psychotraumatol. 8(Suppl. 6):1476441. doi: 10.1080/20008198.2018.1476441

Kim, J., Tol, W. A., Shrestha, A., Kafle, H. M., Rayamajhi, R., Luitel, N. P., et al. (2017). Persistent complex bereavement disorder and culture: early and prolonged grief in Nepali widows. Psychiatry 80, 1-16. doi: 10.1080/00332747. 2016.1213560

Kirmayer, L. J., Jarvis, G. E., and Guzder, J. (2014). "The process of cultural consultation," in Cultural Consultation: Encountering the Other in Mental Health Care, eds L. Kirmayer, G. Jarvis, and J. Guzder, (Berlin: Springer), 47-69. doi: 10.1007/978-1-4614-7615-3-3

Lewis-Fernández, R., Aggarwal, N. K., Lam, P. C., Galfalvy, H., Weiss, M. G., Kirmayer, L. J., et al. (2017). Feasibility, acceptability and clinical utility of the cultural formulation interview: mixed-methods results from the DSM-5 international field trial. Br. J. Psychiatry 210, 290-297. doi: 10.1192/bjp.bp.116. 193862

Li, J., and Prigerson, H. G. (2016). Assessment and associated features of prolonged grief disorder among Chinese bereaved individuals. Compr. Psychiatry 66, 9-16. doi: 10.1016/j.comppsych.2015.12.001

Lobar, S. L., Youngblut, J. M., and Brooten, D. (2006). Cross-cultural beliefs, ceremonies, and rituals surrounding death of a loved one. Peadiatr. Nurs. 32, 44-50.

Maciejewski, P. K., Maercker, A., Boelen, P. A., and Prigerson, H. G. (2016). "Prolonged grief disorder" and "persistent complex bereavement disorder," but not "complicated grief," are one and the same diagnostic entity: an analysis of data from the yale bereavement study. World Psychiatry 15, 266-275. doi: 10.1002/wps.20348

O'Connor, M., Lasgaard, M., Larsen, L., Johannsen, M., Lundorff, M., FarverVestergaard, I., et al. (2019). Comparison of proposed diagnostic criteria for pathological grief using a sample of elderly bereaved spouses in Denmark: perspectives on future bereavement research. J. Affect. Disord. 251, 52-59. doi: 10.1016/j.jad.2019.01.056
China and reviewed the results of the Chinese studies. $\mathrm{CK}$ proposed the idea and directed the whole process of the writing. AM and $\mathrm{M}-\mathrm{FO}{ }^{\prime} \mathrm{C}$ read and revised the manuscript in detail.

Patel, V., Simunyu, E., Gwanzura, F., Lewis, G., and Mann, A. (1997). The Shona Symptom Questionnaire: the development of an indigenous measure of common mental disorders in Harare. Acta Psychiatr. Scand. 95, 469-475. doi: 10.1111/j.1600-0447.1997.tb10134.x

Prigerson, H. G., Horowitz, M. J., Jacobs, S. C., Parkes, C. M., Aslan, M., Goodkin, K., et al. (2009). Prolonged grief disorder: psychometric validation of criteria proposed for DSM-V and ICD-11. PLoS Med. 6:e1000121. doi: 10.1371/journal. pmed.1000121

Prigerson, H. G., and Maciejewski, P. K. (2005). A call for sound empirical testing and evaluation of criteria for complicated grief proposed for DSM-V. Omega 52, 9-19. doi: 10.2190/ANKH-BB2H-D52N-X99Y

Prigerson, H. G., Maciejewski, P. K., Reynolds, C. F. III, Bierhals, A. J., Newsom, J. T., Fasiczka, A., et al. (1995). Inventory of Complicated Grief: a scale to measure maladaptive symptoms of loss. Psychiatry Res. 59, 65-79. doi: 10.1016/ 0165-1781(95)02757-2

Rajkumar, A. P., Mohan, T. S., and Tharyan, P. (2015). Lessons from the 2004 Asian tsunami: nature, prevalence and determinants of prolonged grief disorder among tsunami survivors in South Indian coastal villages. Int. J. Soc. Psychiatry 61, 645-652. doi: 10.1177/0020764015570713

Rasmussen, A., Keatley, E., and Joscelyne, A. (2014). Posttraumatic stress in emergency settings outside North America and Europe: a review of the emic literature. Soc. Sci. Med. 109, 44-54. doi: 10.1016/j.socscimed.2014. 03.015

Shear, M. K., Frank, E., Houck, P. R., and Reynolds, C. F. (2005). Treatment of complicated grief: a randomized controlled trial. JAMA 293, 2601-2608. doi: 10.1001/jama.293.21.2601

Shi, G., Wen, J., Xu, X., Zhou, N., Wang, J., Shi, Y., et al. (2019). Culturerelated grief beliefs of Chinese Shidu parents: development and psychometric properties of a new scale. Eur. J. Psychotraumatol. 10:1626075. doi: 10.1080/ 20008198.2019.1626075

Shoeb, M., Weinstein, H., and Mollica, R. (2007). The Harvard trauma questionnaire: adapting a cross-cultural instrument for measuring torture, trauma and posttraumatic stress disorder in Iraqi refugees. Int. J. Soc. Psychiatry 53, 447-463. doi: 10.1177/0020764007078362

Smid, G. E., Groen, S., de la Rie, S. M., Kooper, S., and Boelen, P. A. (2018). Toward cultural assessment of grief and grief-related psychopathology. Psychiatric Serv. 69, 1050-1052. doi: 10.1176/appi.ps.201700422

Stelzer, E.-M., Zhou, N., Maercker, A., Sun, H., Rohner, S., Merzhvynska, M., et al. (in press). Clinical utility of prolonged grief disorder in the ICD-11: the perspective of Chinese and German-speaking health care professionals (in preparation). Psychopathology.

Tsai, W. I., Kuo, S. C., Wen, F. H., Prigerson, H. G., and Tang, S. T. (2018). Prolonged grief disorder and depression are distinct for caregivers across their first bereavement year. Psychooncology 27, 1027-1034. doi: 10.1002/pon.4629

Tsutsui, T., Hasegawa, Y., Hiraga, M., Ishiki, M., and Asukai, N. (2014). Distinctiveness of prolonged grief disorder symptoms among survivors of the Great East Japan Earthquake and Tsunami. Psychiatry Res. 217, 67-71. doi: 10.1016/j.psychres.2014.03.001

World Health Organization [WHO] (2018). International Statistical Classification of Diseases and Related Health Problems. Geneva: WHO.

Xiu, D., Maercker, A., Woynar, S., Geirhofer, B., Yang, Y., and Jia, X. (2016). Features of prolonged grief symptoms in Chinese and Swiss bereaved parents. J. Nerv. Ment. Dis. 204, 693-701. doi: 10.1097/NMD.000000000000 0539

Yang, B. (1980). Analects of Confucius. Beijing: Zhonghua Book Company.

Yi, X., Gao, J., Wu, C., Bai, D., Li, Y., Tang, N., et al. (2018). Prevalence and risk factors of prolonged grief disorder among bereaved survivors seven years after the Wenchuan earthquake in China: a cross-sectional study. Int. J. Nurs. Sci. 5, 157-161. doi: 10.1016/j.ijnss.2018.04.001

Yin, F., Fang, J., Zhou, N., Shen, H., and He, Y. (2017). Development and validation of the Chinese death metaphors scale-revised. Death Stud. 41, 87-92. doi: 10. 1080/07481187.2016.1214632 
Yu, M., Tang, S., Wang, C., Xiang, Z., Yu, W., Xu, W., et al. (2017). Avoidance of bereavement-related stimuli in Chinese individuals experiencing prolonged grief: evidence from a dot-probe task. Front. Psychol. 8:1201. doi: 10.3389/fpsyg. 2017.01201

Yu, N. X., Chan, C. L., Zhang, J., and Stewart, S. M. (2016). Resilience and vulnerability: prolonged grief in the bereaved spouses of marital partners who died of AIDS. AIDS Care 28, 441-444. doi: 10.1080/09540121.2015.1112354

Zhou, N., Yu, W., Huang, H., Shi, G., Luo, H., Song, C., et al. (2018). Latent profiles of physical and psychological outcomes of bereaved parents in China who lost their only child. Eur. J. Psychotraumatol. 9:1544026. doi: 10.1080/20008198. 2018.1544026
Conflict of Interest: The authors declare that the research was conducted in the absence of any commercial or financial relationships that could be construed as a potential conflict of interest.

Copyright (c) 2020 Stelzer, Zhou, Maercker, O'Connor and Killikelly. This is an openaccess article distributed under the terms of the Creative Commons Attribution License (CC BY). The use, distribution or reproduction in other forums is permitted, provided the original author(s) and the copyright owner(s) are credited and that the original publication in this journal is cited, in accordance with accepted academic practice. No use, distribution or reproduction is permitted which does not comply with these terms. 